\title{
Mindfulness as mediator and moderator of post-traumatic symptomatology in adolescence following childhood sexual abuse
}

\author{
Isabelle Daigneault ${ }^{1}$, Jacinthe Dion ${ }^{2}$, Martine Hébert ${ }^{3}$, Catherine Bourgeois ${ }^{1}$ \\ ${ }^{1}$ Université de Montréal, Psychology Department, P.O. Box 6128, Downtown Station, \\ Montréal, QC, Canada H3C 3J7 \\ ${ }^{2}$ Université du Québec à Chicoutimi, Health Sciences Department, 555 Boulevard de \\ l'Université, Chicoutimi, QC, Canada G7H 2B1 \\ ${ }^{3}$ Université du Québec à Montréal, Sexology Department, P.O. Box 8888, Downtown \\ station, Montréal, QC, Canada H3C 3P8
}

Acknowledgements: The first three authors are members of the "Centre de recherche interdisciplinaire sur les problèmes conjugaux et les agressions sexuelles” (CRIPCAS) and the "Équipe violence sexuelle et santé" (ÉVISSA), both research groups on sexual abuse. This research was supported by a grant from the Université du Québec à Chicoutimi. We thank school directors, teachers and students for their generous collaboration in this project, as well as research assistants, in particular Erika Bacon, Marie-Frédéric Bouchard, François Muckle and Delphine Lagacé for their assistance collecting data.

Corresponding author: Isabelle Daigneault - email: isabelle.daigneault@umontreal.ca, phone: 514-343-2487, fax: 514-343-2285 


\title{
Mindfulness as mediator and moderator of post-traumatic symptomatology in adolescence following childhood sexual abuse or assault
}

\begin{abstract}
Childhood sexual abuse or assault (CSA) is a non-specific risk factor for psychopathology such as post-traumatic stress disorder or depression. However, the impact of CSA greatly varies among individuals. Mediating mechanisms and moderating factors have been proposed to explain how the effects of CSA translate into varied symptoms. Mindfulness is one of the potential variables linked to the diversity of outcomes of CSA. As mindfulness-based interventions are increasingly being used among adolescents and those sexually abused or assaulted in childhood, it is essential to know more about the mechanisms by which it influences post-traumatic symptoms in these populations. This study's aim is to assess whether mindfulness mediates and moderates the relationship between self-reported exposure to CSA and post-traumatic symptoms in adolescence. A sample of 246 adolescents (48\% female) reported their exposure to CSA and completed the Child Acceptance and Mindfulness Measure (Greco et al. 2011) and the Trauma Symptoms Checklist for Children (Briere 1996). The relation between CSA, mindfulness and post-traumatic symptoms was investigated using mediation and moderation regression models from the Process script (Hayes 2013). Results reveal that mindfulness acted as a mediator of post-traumatic symptoms, as CSA was associated with lower levels of mindfulness, which in turn were associated with more post-traumatic symptoms. On the other hand, mindfulness only acted as a moderator of CSA for anger and anxiety, but not in the expected direction. Further investigation is needed to shed light on the moderator role of mindfulness in sexually abused youth.
\end{abstract}


Keywords: Sexual Abuse; Sexaul Assault; Post-Traumatic Stress; Mindfulness; Mediation; Moderation 


\section{Introduction}

Post-traumatic symptomatology associated with childhood sexual abuse or assault (CSA) is diverse as CSA is considered a non-specific risk factor for psychopathology (e.g. depression, anxiety, anger, dissociation, etc.) (Maniglio 2009). A review assessing social and emotional outcomes of CSA among children and adolescents concluded that consequences varied with age, and that adolescents exhibited the greatest variability in mental health problems (e.g., suicidal behaviours, running away, substance abuse) (Tyler 2002).

Adaptive functioning with regards to mental health is also evident in sexually abused or assaulted children and adolescents (Daigneault et al. 2007a; Hébert et al. 2014; Domhardt et al. 2014), which has prompted research on identifying protective factors associated with resilience or adaptive functioning (Marriott et al. 2014; McClure et al. 2008; Spaccarelli and Kim 1995). Indeed, a host of studies suggest that even in the face of severe forms of childhood maltreatment such as sexual abuse or assault, some youth may overcome these traumatic experiences without apparent symptoms (Daigneault et al. 2007b; Kendall-Tackett et al. 1993; Putnam 2003; Hébert et al. 2014). Static factors such as the characteristics of the abusive events have typically been considered in explaining the variability in outcomes presented by youth: the most severe CSA (i.e. chronic abuse, intra-familial, involving penetration) have been associated with elevated prevalence rates of psychiatric disorders (Fergusson et al. 2008; Fergusson et al. 1996). However, these factors have not always yielded unequivocal results in predicting outcomes in sexually abused or assaulted youth (Brabant et al. 2014). In addition, CSA severity is not amenable to change in treatment. To foster resilience and forestall psychopathology, researchers have begun to study processes that may transmit the detrimental effect of CSA (mediator) as well as protective factors that may act as buffers (moderator) for negative outcomes in children, 
adolescents (Daigneault et al. 2007b; Daigneault et al. 2007a; Spaccarelli and Kim 1995) and adult survivors (Sperry and Widom 2013).

Indeed, some mechanisms through which the detrimental effects of CSA translate into symptoms, i.e. mediators, have been proposed to explain the varied consequences found in the scientific literature (Finkelhor and Browne 1985; Spaccarelli 1994). In one of the first proposals to this effect, Spaccarelli (1994) has argued that CSA will limit children's resources, which in turn will entail elevated symptoms. The more a child's resources are affected by the CSA, the more one can expect the child will show post-traumatic symptoms. For example, results of a study on sexually abused and non-abused preschoolers have indicated that emotional regulation abilities mediated the relationship between sexual abuse and internalized or externalized difficulties (Langevin et al. 2015). That is, sexually abused children were less able to regulate their emotions than their non-abused counterparts, which in turn predicted their more elevated symptoms.

In turn, the moderator or protective factor model suggests that when a protective factor is present, it mitigates the negative effect of the abuse on youth in such a way that they exhibit less post-traumatic symptoms or deficits than their peers who present lower levels of the studied protective factor, but similar levels of exposure to the risk factor (Luthar et al. 2000). Previous studies have revealed that a number of protective factors can moderate the negative consequences of CSA (e.g., empowerment, interpersonal trust; Daigneault et al. 2007b).

Among potential variables linked to the diversity of outcomes, recent clinical and research endeavours have focused on mindfulness. Being able to pay attention to novelty and to the context of a current situation (Langer et al. 1978) or in a purposeful manner, in the present moment, and non-judgmentally (Kabat-Zinn et al. 1985), is thought to help reduce 
psychopathology in general and post-traumatic symptoms, in particular (Banks et al. 2015; Follette et al. 2006). In this regard, mindfulness can also be conceptualized as at least partly countering experiential avoidance (Thompson and Waltz 2010). Interventions have thus been developed to help enhance this protective factor and increase mindfulness abilities whose development may have been thwarted by CSA. Mindfulness-based interventions, such as Acceptance and Commitment Therapy (ACT) (A-Tjak et al. 2015; Ost 2014), are among those that are rapidly gaining in popularity to help decrease a variety of mental health problems in children and adolescents (Tovote et al. 2014; Broderick and Jennings 2012; Cotton et al. 2015; Zoogman et al. 2015), as well as in children, adolescents and adult survivors of childhood maltreatment (Kimbrough et al. 2010; Caldwell and Shaver 2015; Lanktree et al. 2012; Mannarino et al. 2012). Mindfulness training has been found to help diminish symptoms of depression, anxiety, post-traumatic stress disorder (PTSD), sexual dysfunction and general distress in adult women reporting CSA (Earley et al. 2014; Kimbrough et al. 2010; Brotto et al. 2012) and to reduce behavioural, externalized and internalized problems among youth reporting various types of childhood maltreatment (Swart and Apsche 2014).

Although many studies have been conducted on the effectiveness of mindfulness interventions, few have investigated mindfulness as a mediator or a moderator in the association between sexual abuse and its sequelae. One recent study has found that cumulative traumatic experiences in childhood, including sexual abuse, were associated with depression in adults consulting for sex therapy, and that mindfulness and dissociation mediated that relationship (Bolduc et al. 2014). Thus, adults exposed to a greater number of traumatic experiences in childhood exhibited lower levels of mindfulness than their less exposed counterparts, which in turn was associated with more symptoms of depression. Another indication that a lack of 
emotional mindfulness may carry the detrimental effects of CSA is observed when avoidance of internal emotional experiences mediates the relationship between severity of CSA and psychological distress in adult women sexually abused in childhood (Rosenthal et al. 2005). In other words, these results indicated that more severe abuses predicted more avoidance of internal experiences, which in turn was associated with greater distress.

With regards to mindfulness as a potential protective factor, we have found no study indicating that a greater capacity for mindful emotional and cognitive awareness mitigates the effect of CSA. However, studies on the effect of daily stress have shown that mindfulness acted as a buffer or moderator protecting youth from dysphoria (Ciesla et al. 2012), anxiety and depression (Marks et al. 2010). Indeed, results revealed that at similar levels of stress, higher levels of mindfulness predicted less dysphoria in male youth when compared to those with lower levels of mindfulness (Ciesla et al. 2012). Study results have also shown that higher levels of daily stress in high-school male and female youth were associated with more symptoms of depression and anxiety, but only for those that had low levels of mindfulness (Marks et al. 2010). Youth exhibiting high levels of mindfulness seemed to be protected against the effects of daily stress, at least with regards to depression and anxiety.

To our knowledge, only one study has assessed the dual mediator/moderator role of mindfulness. This study was conducted with young adults and predicted internalizing symptoms according to their exposure to childhood emotional maltreatment (English 2012). Results of this dissertation revealed that participants with greater emotional maltreatment exposure had more attachment disturbances in the form of avoidance, which in turn was associated with lower mindfulness, which predicted more internalizing symptoms. In addition, mindfulness moderated the effect of exposure to childhood emotional maltreatment with regards to internalizing 
symptoms in such a way that only those with low levels of mindfulness (one standard deviation below the mean) exhibited more internalizing symptoms when exposed to childhood emotional maltreatment.

Because mindfulness-based interventions are increasingly being used with youth and with those exposed to childhood trauma such as sexual abuse (Follette et al. 2015), it remains important to document mindfulness’ relationship to childhood traumatic experiences and their consequences. Such information is currently lacking for sexually abused or assaulted adolescent populations, and further studies will provide relevant cues for the design of interventions.

The goal of the current study is to assess whether mindfulness mediates and moderates the relationship between self-reported exposure to sexual abuse or assault and post-traumatic symptoms in adolescence (anxiety, depression, PTSD, sexual concerns, dissociation and anger). Consistent with previous findings, the study's hypotheses are twofold: 1) we expect a significant indirect effect of CSA on levels of anxiety, depression, PTSD, sexual concerns, dissociation and anger in youth that will be exerted through mindfulness (mediation hypothesis). 2) Despite the fact that mindfulness is thought to be negatively affected by CSA, which entails more symptoms (mediation hypothesis), other factors may be contributing to current levels of mindfulness in both sexually abused or assaulted and non-exposed youth (e.g., parents' mindfulness, mindfulness practice or intervention, etc.). This may act as a buffer against the effect of CSA on symptoms. We thus expect that the relationship between CSA and post-traumatic symptoms will be stronger for participants with low mindfulness scores and weaker or non-significant for participants with high mindfulness scores, as indicated by statistically significant interactions between CSA and mindfulness in predicting post-traumatic symptoms (moderation hypothesis).

\section{Method}




\section{Participants}

The sample of 245 adolescents was drawn from one high-school in Saguenay-Lac-StJean (Québec province, Canada). Participants represent 92\% of the targeted sample (8\% absentees, missing data and invalid protocols). Adolescents were aged between 14 and 19 years (grades $9-11$ and $M=15.89$ years, $S D=0.95)$, and 47,3\% $(n=116)$ were female. The majority of participants’ parents had at least a high-school diploma (79.9\% of mothers, $71.9 \%$ of fathers). The majority of adolescents lived with their two biological parents (56.4\%), whereas 25.1\% lived in a single-parent household or in a joint custody setting, $14.4 \%$ lived in a two-parent stepfamily, and $4.1 \%$ in an adopted family or with another family member (e.g., grandparent).

\section{Procedures}

The study received ethical approval from Université du Québec à Chicoutimi. A rural high-school was selected to take part in a larger study on youth resilience (Daigneault, Dion, Hébert, McDuff, Collin-Vézina, 2013; Muckle, Dion, Daigneault, Ross, \& McDuff, 2012). A consent form was distributed to each student in selected classrooms, which explained the research modalities. Each participating student then received an anonymous questionnaire that did not enable participant identification. Participants completed questionnaires in the school library two groups/classes of students at a time, which took on average 30 minutes. Research assistants responsible for recruitment and data collection were available to answer questions and to ensure students could complete questionnaires in confidentiality. All enrolled students present the day of assessment accepted to participate and completed the in-class survey.

\section{Measures}

Childhood Sexual Abuse or Assault. Youths’ experiences of sexual abuse or assault were assessed with one item concerning all unwanted sexual contact occurring before the study 
with a variety of perpetrators: “The next item represents a situation that might have happened to you with different people (family member, date, romantic partner, friend, neighbour, coach, acquaintance, stranger, etc.). Have you ever had a sexual relationship, were subjected to sexual behaviours or were forced to behave sexually with one of these people while you did not want to?” Youth answering “Yes” were coded as being sexually abused or assaulted, while those answering "No” were coded as not being sexually abused or assaulted. This item has been used in previous studies and yielded CSA prevalence rates (12 to 13\%) (Daigneault, Dion, Hébert, McDuff, \& Collin-Vézina, 2013) similar to those of recent meta-analyses (Stoltenborgh et al. 2011; Barth et al. 2013).

Mindfulness. The 10-item version of the Child self-Acceptance, Mindfulness Measure (CAMM - Greco et al., 2011), was used to assess self-acceptance/mindfulness in adolescents. Items are scored on a five-point Likert scale ranging from $0=$ never true to $4=$ always true. Scores range from 0 to 40, with higher scores indicating greater abilities for present-moment awareness and non-judgmental accepting responses to thoughts and feelings. The CAMM has been validated for use with non-clinical adolescent populations (de Bruin et al. 2014; Greco et al. 2011; Kuby et al. 2015).

Post-Traumatic Outcomes. Trauma symptoms were assessed using the Trauma Symptoms Checklist for Children (TSCC - Briere, 1996), a 54-item questionnaire for youth 8 to 17 years of age scored on a four-point Likert scale from $0=$ never to $3=$ almost always. Subscales enable to assess symptoms of depression, anxiety, PTSD, sexual concerns, dissociation, and anger. Higher scores denote more symptoms in each subscale (Briere, 1996). Because TSCC scores vary according to gender (Martinez et al. 2014), gender was controlled for in all analyses. 


\section{Data Analyses}

To test whether data were consistent with mediation and moderation hypotheses, multiple ordinary least-squares (OLS) regressions were computed using model 4 from PROCESS (Figure 1) (Hayes 2013) with IBM SPSS version 22. Indirect mediation effects were formally and directly tested using 10000 resampling bias-corrected bootstrap confidence intervals (CI). They were considered statistically significant when 0 was excluded from the interval. Moderation effects were assessed using normal theory tests of significance using model 1 from Process (Hayes 2013). All analyses used a 95\% level of confidence.

\section{Results}

Table 1 provides descriptive statistics and correlations for the study’s variables. Results reveal that close to $14 \%$ of adolescents reported CSA (19\% for females, and $9 \%$ for males). The participants' total mindfulness score $(M=26.05$, range 7 to 40$)$ indicates that they were on average "sometimes” to "often” mindful, with 31\% being often or always mindfully aware and accepting of their thoughts and emotions and only $2 \%$ being on average never or rarely mindfully aware and accepting. Outcome measures yield rates of post-traumatic symptoms reaching clinical significance that vary from 3\% (anger) to 25\% (sexual concerns). There is no evidence of floor or ceiling effect problems as less than 15\% of participants have the lowest or highest possible scores on all measures (Terwee et al. 2007).

Results presented in Table 2 show regression coefficients for the direct effect of CSA on mindfulness scores (path a), on six posttraumatic outcomes (path c'), the effect of mindfulness on six post-traumatic outcomes (path b) and the indirect effect of CSA on six post-traumatic outcomes through mindfulness (path ab; all paths are illustrated in Figure 1). Statistically significant positive coefficients for paths ab (all confidence intervals exclude 0 ) indicate that 
CSA was indirectly associated with five post-traumatic outcomes through mindfulness, which acted as a mediator. The coefficient of determination $\left(R^{2}\right)$ indicates that the mediation models explained between 18\% (Anger) and 35\% (PTSD) of the variance in outcomes. These significant mediation models indicate that sexually abused adolescents reported lower levels of mindfulness than their non-abused counterparts, which in turn explained why they also reported more symptoms of anxiety, depression, PTSD, sexual concerns, dissociation, and anger. The ratio of indirect to total effect of CSA on the six post-traumatic outcomes indicates that mindfulness carried between $9 \%$ and $25 \%$ of the sexual abuse or assault's total effect, depending on outcomes.

Results presented in Table 3 indicate that mindfulness moderates CSA's association with anxiety and anger only. This means that youth's anxiety and anger are not affected similarly by CSA status and that this effect depends on youth's level of mindfulness. Figure 2 illustrates these two statistically significant interactions using the pick-a-point and Johnson-Neyman approaches (Hayes and Matthes 2009; Miller et al. 2013; Bauer and Curran 2005). We first calculated predicted levels of anxiety and anger at five predetermined levels of mindfulness $\left(10^{\text {th }}, 25^{\text {th }}, 50^{\text {th }}\right.$, $75^{\text {th }}$, and $90^{\text {th }}$ percentiles). This yielded the illustrated slopes for the CSA group and the non-CSA group and indicated that the two groups had average levels of anxiety and anger that were statistically different at higher levels of Mindfulness only $\left(50^{\text {th }}, 75^{\text {th }}\right.$ and $90^{\text {th }}$ percentiles $)$. We further calculated the exact mindfulness level at which the relationship between CSA and anxiety and anger ceased to be statistically significant. This is illustrated in Figure 2 by the JohnsonNeyman cut points and indicates that CSA was associated with higher levels of anxiety and anger only for youth with mindfulness scores above 21.27 and 20.13, respectively. This confirms that at below-average levels of mindfulness ( $25^{\text {th }}$ percentile and below), abused and non-abused 
youth exhibited similar levels of anxiety and anger. The lack of statistically significant interactions between CSA and mindfulness in predicting all other post-traumatic outcomes indicates that CSA is similarly associated with depression, PTSD, sexual concerns and dissociation scores at all levels of mindfulness.

\section{Discussion}

The goal of the current study was to determine whether mindfulness mediated and moderated the relationship between CSA and post-traumatic symptoms in adolescents. The results partially support the study's hypotheses. As predicted, mindfulness explained part of CSA's effect on post-traumatic symptomatology in youth, acting as a mediator between CSA and outcomes. Indeed, those exposed to CSA had lower mindfulness scores, which in turn predicted their more elevated symptom scores in anxiety, depression, PTSD, sexual concerns, dissociation and anger. Mindfulness acted as a moderator of the relationship between CSA and two of the six outcomes considered, anxiety and anger, but not in the predicted direction.

The results of the mediation analyses confirm the study's first hypothesis and are in line with results of previous studies with adult populations exposed to CSA or to cumulative traumatic experiences that include CSA (Bolduc et al. 2014; Rosenthal et al. 2005). Results of the current study thus give further evidence that CSA entails a lessened ability to be aware of thoughts and feelings in the present-moment and to observe these thoughts and feelings with acceptance and without judgment in adolescents. These lessened abilities are part of the mechanisms by which sexually abused adolescents suffer from more post-traumatic symptoms, such as depression, anxiety, PTSD, sexual concerns, dissociation, and anger.

Because it seems to be one of the mechanisms through which CSA is detrimental to one's psychological functioning, interventions aimed at increasing one's ability to be mindfully aware 
of thoughts and emotions and accepting could be effective for reducing sexually abused youth’s post-traumatic symptoms. Recent meta-analyses have revealed some of the active ingredients in mindfulness-based cognitive and stress reduction treatments (Gu et al. 2015; van der Velden et al. 2015). They have shown that adult participants decrease their cognitive and emotional reactivity, rumination and worry and increase their mindfulness, their self-compassion and their meta-awareness, which are in turn associated with decreases in depression (van der Velden et al. 2015), anxiety, global psychopathological symptoms, stress and negative affect (Gu et al. 2015).

The results of the moderation analyses, however, do not confirm the study's second hypothesis. If mindfulness acts as a statistically significant moderator of the relationship between CSA, and the outcomes of anxiety and anger, the moderation is not in the expected direction. Based on previous study results, we hypothesized that sexually abused youth with elevated levels of mindfulness would be "protected” against the detrimental effects of CSA and would thus show less post-traumatic symptoms at higher levels of mindfulness, or even no effect of CSA at those higher levels. Previous studies of the effect of daily stress (Ciesla et al. 2012; Marks et al. 2010) and childhood emotional maltreatment (English 2012) on internalizing symptoms such as dysphoria, anxiety and depression have indeed shown that daily stress and emotional maltreatment were only detrimental at low mindfulness levels. Participants exhibiting high levels of mindfulness did not show as many symptoms when similarly exposed to stress or emotional maltreatment as their peers with lower levels of mindfulness. On the contrary, results of the current study reveal that it is at the lowest levels of mindfulness that CSA has no effect on anxiety and anger, because sexually abused youth do not differ from their non-abused counterparts in their levels of anxiety and anger when their mindfulness level is below the $25^{\text {th }}$ percentile. When the interpretation of the results focuses on non-abused youth, it seems that very 
low levels of mindfulness is a risk factor for them developing more anxiety and anger at levels that are undistinguishable from those of their sexually abused counterparts.

These results are surprising and may be partly explained by differences in the type and level of exposure to stress (CSA vs. daily stress or emotional maltreatment) and characteristics of the selected samples (age, socioeconomic level). Although unexpected, results of the current study are not exceptional. Other studies have found that higher levels of acceptance vis-à-vis one’s thoughts and emotions were associated with higher levels of physical partner violence perpetration (Godbout et al., 2016), that under certain conditions, higher levels of self-kindness were associated with increases in anxiety (Valdez and Lilly 2015) and that mindfulness exercises focused on bodily sensations may increase anxiety and treatment dropout, especially for those with a history of sexual abuse (King et al. 2013). With regards to our study results, it may be that initially, being more aware in a context of sexual abuse is associated with experiencing more anxiety and anger. For sexually abused children, dissociation may serve to maintain the relationship with the abusing caregiver and thus to repress anger or memories of the abuse and lessen anxiety (Herman 1992). Becoming aware of one’s emotions and cognitions, less dissociated, may thus initially entail a resurgence of suppressed anger and anxiety. Anger may also initially be viewed as enabling victims to become empowered (Rogers et al. 1997; Wowra and McCarter 1999). Mindfulness’ buffering effect seen in other studies may become evident at a later stage of recovery, once sexually abused adolescents are safer and able to regulate intense emotions.

It is also surprising to observe that mindfulness has no moderating effect on other symptoms, such as depression and PTSD. However, because this is to our knowledge the first study to assess the protective/moderator role of mindfulness regarding post-traumatic symptoms 
in the context of CSA, these results need to be replicated. Mindfulness may act as a protective factor differently, depending on the type of adverse experience. Indeed, mindfulness has been found to moderate the effect of daily stress on depression (Marks et al. 2010) and dysphoria (Ciesla et al. 2012), as well as the effect of childhood emotional maltreatment on internalizing symptoms in general (English 2012).

The combined results indicate that sexually abused youth report lower mindfulness levels than their non-abused counterparts, which explains why they also report more post-traumatic symptoms. However, when sexually abused youth manage to develop moderate to high levels of mindfulness it is associated with higher levels of anxiety and anger in comparison to sexually abused youth that have very low mindfulness levels (below $25^{\text {th }}$ percentile). The mediation effect showing that low levels of mindfulness were associated with high levels of anxiety and anger, seemed to have been mostly attributable to the non-abused participants, since this relationship is reversed for sexually abused participants.

\section{Recommendations}

The current study's inclusion of adolescent males and females in testing the mediation and moderation effects of mindfulness provides preliminary evidence of the relationships among

the studied variables that need to be replicated. Among the limitations that need to be considered, the fact that the study uses cross-sectional data precludes any conclusion regarding the temporal effects of CSA and mindfulness on post-traumatic symptoms. Our use of a global measure of mindfulness also precludes assessing whether different aspects are uniquely associated with traumatic symptoms. This is important to consider in future studies as one study found that it was mostly high levels of the non-judging component of mindfulness, but not the observing and acting with awareness components, that predicted less anger rumination and, in turn, less anger 
and aggression (Peters et al. 2015). Other mindfulness-related constructs not assessed in the current study (e.g. attentional capacity, dissociation, impulsivity, affective self-regulation, etc.), may also act as mediators and/or moderators of post-traumatic outcomes in sexually abused youth and need to be considered alongside mindfulness. In addition, our study results may only be generalized to rural youth populations from low socioeconomic settings. The results need to be replicated in varied populations to determine whether the relationships are affected by socioeconomic and living conditions.

Although mindfulness-based interventions for youth have not been associated with iatrogenic effects (Zoogman et al. 2015), further studies need to document their effectiveness in reducing post-traumatic symptoms in sexually abused youth before they can be safely recommended for these vulnerable populations. Indeed, up until now, these interventions have mostly been assessed in generally healthy youth populations, although they seem to be most effective for reducing psychological symptoms in clinical populations (Zoogman et al. 2015). Effectiveness studies need to be conducted over increased periods of time because improving youth's ability to be mindfully aware of their thoughts and emotions may initially be associated with increases in anxiety and anger in sexually abused youth and has entailed increases in anxiety or aggressive behaviours in other populations (Godbout et al. 2016; King et al. 2013; Valdez and Lilly 2015). In this regard, mindfulness-based interventions initially teaching stress reduction/emotional regulation strategies may be most appropriate to mitigate potentially distressing effects for sexually abused youth (Briere and Scott 2015; Daigneault et al. 2004; Lebowitz et al. 1993).

\section{Compliance with Ethical Standards}

Funding: This study was funded by a grant from the Université du Québec à Chicoutimi. 
Conflict of interest: The authors declare that they have no conflict of interest. 


\section{References}

A-Tjak, J. G., Davis, M. L., Morina, N., Powers, M. B., Smits, J. A., \& Emmelkamp, P. M. (2015). A meta-analysis of the efficacy of acceptance and commitment therapy for clinically relevant mental and physical health problems. Psychotherapy and Psychosomatics, 84(1), 30-36, doi:10.1159/000365764.

Banks, K., Newman, E., \& Saleem, J. (2015). An overview of the research on mindfulness-based interventions for treating symptoms of posttraumatic stress disorder: A systematic review. Journal of Clinical Psychology, Online prepublication, doi:http://dx.doi.org/10.1002/jclp.22200.

Barth, J., Bermetz, L., Heim, E., Trelle, S., \& Tonia, T. (2013). The current prevalence of child sexual abuse worldwide: a systematic review and meta-analysis. International Journal of Public Health, 58(3), 469-483, doi:10.1007/s00038-012-0426-1.

Bauer, D. J., \& Curran, P. J. (2005). Probing interactions in fixed and multilevel regression: Inferential and graphical techniques. Multivariate Behavioral Research, 40(3), 373-400, doi:10.1207/s15327906mbr4003_5.

Bolduc, R., Bigras, N., Godbout, N., Hébert, M., \& Daspe, M.-È. (2014). L'effet de la pleine conscience et de la dissociation sur la relation entre le trauma cumulatif et la dépression : Modèle d'équations structurelles. Paper presented at the Rencontre annuelle du CRIPCAS, Trois-Rivièves, October 2014

Brabant, M.-E., Hébert, M., \& Chagnon, F. (2014). Predicting Suicidal Ideations in Sexually Abused Female Adolescents: A 12-Month Prospective Study. Journal of Child Sexual Abuse, 23(4), 387-397, doi:10.1080/10538712.2014.896842. 
Briere, J. (1996). Trauma Symptom Checklist for Children (TSCC): Professional manual. Odessa: Psychological Assessment Resources, Inc.

Briere, J., \& Scott, C. (2015). Complex Trauma in Adolescents and Adults. Psychiatric Clinics, 38(3), 515-527, doi:10.1016/j.psc.2015.05.004.

Broderick, P. C., \& Jennings, P. A. (2012). Mindfulness for adolescents: a promising approach to supporting emotion regulation and preventing risky behavior. New directions for youth development, 2012(136), 111-126, 111, doi:10.1002/yd.20042.

Brotto, L. A., Seal, B. N., \& Rellini, A. (2012). Pilot study of a brief cognitive behavioural versus mindfulness-based intervention for women with sexual distress and a history of childhood sexual abuse. Journal of Sex \& Marital Therapy, 38(1), 1-27, doi:http://dx.doi.org/10.1080/0092623X.2011.569636.

Caldwell, J. G., \& Shaver, P. R. (2015). Promoting attachment-related mindfulness and compassion: a wait-list-controlled study of women who were mistreated during childhood. Mindfulness, 6(3), 624-636, doi:10.1007/s12671-014-0298-y.

Ciesla, J., Reilly, L., Dickson, K., Emanuel, A., \& Updegraff, J. (2012). Dispositional mindfulness moderates the effects of stress among adolescents: Rumination as a mediator. Journal of Clinical Child and Adolescent Psychology, 41(6), 760-770, doi:http://dx.doi.org/10.1080/15374416.2012.698724.

Cotton, S., Luberto, C. M., Sears, R. W., Strawn, J. R., Stahl, L., Wasson, R. S., et al. (2015). Mindfulness-based cognitive therapy for youth with anxiety disorders at risk for bipolar disorder: a pilot trial. Early Intervention in Psychiatry, 1-9, doi:10.1111/eip.12216. 
Daigneault, I., Cyr, M., \& Tourigny, M. (2007a). Exploration of recovery trajectories in sexually abused adolescents. Journal of Aggression Maltreatment and Trauma, 14(1/2), 165-184, doi:http://dx.doi.org/10.1300/J146v14n01_09.

Daigneault, I., Dion, J., Hébert, M., McDuff, P., \& Collin-Vézina, D. (2013). Psychometric properties of the Child and Youth Resilience Measure (CYRM-28) among samples of French Canadian youth. Child Abuse and Neglect, 37, 160-171. doi: http://dx.doi.org/10.1016/j.chiabu.2012.06.004

Daigneault, I., Hébert, M., \& Tourigny, M. (2007b). Personal and interpersonal characteristics related to resilient developmental pathways of sexually abused adolescents. Child and Adolescent Psychiatric Clinics of North America, 16(2), 415-434, doi:http://dx.doi.org/10.1016/j.chc.2006.11.002.

Daigneault, I., Tourigny, M., \& Cyr, M. (2004). Description of trauma and resilience in sexually abused adolescents: An integrated assessment. Journal of Trauma Practice, 3(2), 23-47. de Bruin, E. I., Zijlstra, B. J. H., \& Bogels, S. M. (2014). The Meaning of Mindfulness in Children and Adolescents: Further Validation of the Child and Adolescent Mindfulness Measure (CAMM) in Two Independent Samples from The Netherlands. Mindfulness, 5(4), 422-430, doi:10.1007/s12671-013-0196-8.

Domhardt, M., Münzer, A., Fegert, J. M., \& Goldbeck, L. (2014). Resilience in survivors of child sexual abuse : A systematic review of the litterature. Trauma, Violence, \& Abuse, 118, doi:10.1177/1524838014557288.

Earley, M. D., Chesney, M. A., Frye, J., Greene, P. A., Berman, B., \& Kimbrough, E. (2014). Mindfulness intervention for child abuse survivors: A 2.5-year follow-up. Journal of Clinical Psychology, 70(10), 933-941, doi:http://dx.doi.org/10.1002/jclp.22102. 
English, L. (2012). Investigating childhood emotional maltreatment, adult attachment, and mindfulness as predictors of internalizing symptoms and emotional processing. University of Guelph, Ontario, Canada.

Fergusson, D. M., Boden, J. M., \& Horwood, L. (2008). Exposure to childhood sexual and physical abuse and adjustment in early adulthood. Child Abuse \& Neglect, 32(6), 607619, doi:http://dx.doi.org/10.1016/j.chiabu.2006.12.018.

Fergusson, D. M., Horwood, J. L., \& Lynskey, M. T. (1996). Childhood sexual abuse and psychiatric disorder in young adulthood: II. psychiatric outcomes of childhood sexual abuse. Journal of American Academy of Child \& Adolescent Psychiatry, 34(10), 13651374.

Finkelhor, D., \& Browne, A. (1985). The traumatic impact of child sexual abuse: A conceptualization. American Journal of Orthopsychiatry, 55(4), 530-541.

Follette, V. M., Briere, J., Rozelle, D., Hopper, J. W., \& Rome, D. I. (2015). Mindfulnessoriented interventions for trauma: Integrating contemplative practices. In Mindfulnessoriented interventions for trauma: Integrating contemplative practices (pp. xi, 372). New York, NY: Guilford Press; US.

Follette, V. M., Palm, K. M., \& Pearson, A. N. (2006). Mindfulness and trauma: Implications for treatment. Journal of Rational Emotive \& Cognitive Behavior Therapy, 24(1), 45-61, doi:http://dx.doi.org/10.1007/s10942-006-0025-2.

Godbout, N., Bigras, N., \& Dion, J. (2016). Présence attentive et traumas interpersonnels en enfance [Mindfulness and interpersonnal trauma in childhood]. In S. Grégoire, L. Lachance, \& L. Richer (Eds.), La présence attentive : état des connaissances théoriques, 
empiriques et pratiques (pp. 229-246). [Mindfulness: Current theoretical, empirical and practical knowledge]. Montreal: Presses de l’Université du Québec (PUQ).

Greco, L. A., Baer, R. A., \& Smith, G. T. (2011). Assessing mindfulness in children and adolescents: Development and validation of the Child and Adolescent Mindfulness Measure (CAMM). Psychological Assessment, 23(3), 606-614, doi:http://dx.doi.org/10.1037/a0022819.

Gu, J., Strauss, C., Bond, R., \& Cavanagh, K. (2015). How do mindfulness-based cognitive therapy and mindfulness-based stress reduction improve mental health and wellbeing? A systematic review and meta-analysis of mediation studies. Clinical Psychology Review, 37, 1-12, doi:10.1016/j.cpr.2015.01.006.

Hayes, A. F. (2013). Introduction to Mediation, Moderation and Conditional Process Analysis : A Regression-Based Approach (Vol. 1 april 2014). New York, US: The Guildford Press. Hayes, A. F., \& Matthes, J. (2009). Computational procedures for probing interactions in OLS and logistic regression: SPSS and SAS implementations. Behavior research methods, 41(3), 924-936.

Hébert, M., Lavoie, F., \& Blais, M. (2014). Post Traumatic Stress Disorder/PTSD in adolescent victims of sexual abuse: resilience and social support as protection factors. Ciência \& Saúde Coletiva, 19(3), 685-694.

Herman, J. L. (1992). Trauma and Recovery. New-York: Basic Books.

Kabat-Zinn, J., Lipworth, L., \& Burney, R. (1985). The clinical use of mindfulness meditation for the self-regulation of chronic pain. Journal of Behavioral Medicine, 8(2), 163-190. 
Kendall-Tackett, K. A., Williams, L. M., \& Finkelhor, D. (1993). Impact of sexual abuse on children: A review and synthesis of recent empirical studies. Psychological Bulletin, 113(1), 164-180, doi:http://dx.doi.org/10.1037/0033-2909.113.1.164.

Kimbrough, E., Magyari, T., Langenberg, P., Chesney, M., \& Berman, B. (2010). Mindfulness intervention for child abuse survivors. Journal of Clinical Psychology, 66(1), 17-33.

King, A. P., Erickson, T. M., Giardino, N. D., Favorite, T., Rauch, S. A. M., Robinson, E., et al. (2013). A pilot study of group mindfulness-based cognitive therapy (MBCT) for combat veterans with posttraumatic stress disorder (PTSD). Depression and Anxiety, 30(7), 638645, doi:10.1002/da.22104.

Kuby, A., McLean, N., \& Allen, K. (2015). Validation of the Child and Adolescent Mindfulness Measure (CAMM) with Non-Clinical Adolescents. Mindfulness, 1-8, doi:10.1007/s12671-015-0418-3.

Langer, E. J., Blank, A., \& Chanowitz, B. (1978). The mindlessness of ostensibly thoughtful action: The role of" placebic" information in interpersonal interaction. Journal of Personality and Social Psychology, 36(6), 635.

Langevin, R., Hébert, M., \& Cossette, L. (2015). Emotion regulation as a mediator of the relation between sexual abuse and behavior problems in preschoolers. Child Abuse \& Neglect, 46, 16-26, doi:http://dx.doi.org/10.1016/j.chiabu.2015.02.001.

Lanktree, C. B., Briere, J., Godbout, N., Hodges, M., Chen, K., Trimm, L., et al. (2012). Treating Multitraumatized, Socially Marginalized Children: Results of a Naturalistic Treatment Outcome Study. Journal of Aggression, Maltreatment \& Trauma, 21(8), 813-828, doi:10.1080/10926771.2012.722588. 
Lebowitz, L., Harvey, M. R., \& Herman, J. L. (1993). A stage-by-dimension model of recovery from sexual trauma. Journal of Interpersonal Violence, 8(3), 378-391.

Luthar, S. S., Cicchetti, D., \& Becker, B. (2000). The construct of resilience: A critical evaluation and guidelines for future work. Child Development, 71(3), 543-562, doi:http://dx.doi.org/10.1111/1467-8624.00164.

Maniglio, R. (2009). The impact of child sexual abuse on health: A systematic review of reviews. Clinical Psychology Review, 29(7), 647-657, doi:http://dx.doi.org/10.1016/j.cpr.2009.08.003.

Mannarino, A. P., Cohen, J. A., Deblinger, E., Runyon, M. K., \& Steer, R. A. (2012). Traumafocused Cognitive-Behavioral Therapy for children: Sustained impact of treatment 6 and 12 months later. Child Maltreatment, 17(3), 231-241, doi:http://dx.doi.org/10.1177/1077559512451787.

Marks, A. D. G., Sobanski, D. J., \& Hine, D. W. (2010). Do dispositional rumination and/or mindfulness moderate the relationship between life hassles and psychological dysfunction in adolescents? Australian and New Zealand Journal of Psychiatry, 44(9), 831-838.

Marriott, C., Hamilton-Giachritsis, C., \& Harrop, C. (2014). Factors promoting resilience following childhood sexual abuse: A structured, narrative review of the literature. Child Abuse Review, 23(1), 17-34.

Martinez, W., Polo, A. J., \& Zelic, K. J. (2014). Symptom variation on the trauma symptom checklist for children: A within-scale meta-analytic review. Journal of Traumatic Stress, 27(6), 655-663, doi:http://dx.doi.org/10.1002/jts.21967. 
McClure, F. H., Chavez, D. V., Agars, M. D., Peacock, M., \& Matosian, A. (2008). Resilience in sexually abused women: Risk and protective factors. Journal of Family Violence, 23(2), 81-88, doi:http://dx.doi.org/10.1007/s10896-007-9129-4.

Miller, J. W., Stromeyer, W. R., \& Schwieterman, M. A. (2013). Extensions of the JohnsonNeyman technique to linear models with curvilinear effects: Derivations and analytical tools. Multivariate Behavioral Research, 48(2), 267-300.

Muckle, F., Dion, J., Daigneault, I., Ross, A., \& McDuff, P. (2012). Influence de l'estime de soi, de la perception des qualités relationnelles parents-enfants, du soutien social et de l’agression sexuelle sur la résilience auprès d’adolescents autochtones et caucasiens du Québec [Influence of self-esteem, parent-child relationships, social support and sexual abuse on resilience among Aboriginal and Caucasian adolescents]. First Peoples Child \& Family Review, 7. 99-117.

Ost, L. G. (2014). The efficacy of Acceptance and Commitment Therapy: an updated systematic review and meta-analysis. Behav Res Ther, 61, 105-121, doi:10.1016/j.brat.2014.07.018.

Peters, J. R., Smart, L. M., Eisenlohr-Moul, T. A., Geiger, P. J., Smith, G. T., \& Baer, R. A. (2015). Anger rumination as a mediator of the relationship between mindfulness and aggression: The utility of a multidimensional mindfulness model. Journal of Clinical Psychology, n/a-n/a, doi:10.1002/jclp.22189.

Putnam, F. W. (2003). Ten-year research update review: Child sexual abuse. Journal of the American Academy of Child \& Adolescent Psychiatry, 42(3), 269-278, doi:10.1097/00004583-200303000-00006. 
Rogers, E. S., Chamberlin, J., Ellison, M. L., \& Crean, T. (1997). A consumer-constructed scale to measure empowerment among users of mental health services. Psychiatric Services, 48(8), 1042-1047.

Rosenthal, M. Z., Hall, M. L. R., Palm, K. M., Batten, S. V., \& Follette, V. M. (2005). Chronic avoidance helps explain the relationship between severity of childhood sexual abuse and psychological distress in adulthood. Journal of Child Sexual Abuse, 14(4), 25-41, doi:10.1300/J070v14n04_02.

Spaccarelli, S. (1994). Stress, appraisal, and coping in child sexual abuse: A theoretical and empirical review. Psychological Bulletin, 116(2), 340-362.

Spaccarelli, S., \& Kim, S. (1995). Resilience criteria and factors associated with resilience in sexually abused girls. Child Abuse \& Neglect, 19(9), 1171-1182, doi:http://dx.doi.org/10.1016/0145-2134\%2895\%2900077-L.

Sperry, D. M., \& Widom, C. S. (2013). Child abuse and neglect, social support, and psychopathology in adulthood: a prospective investigation. Child Abuse \& Neglect, 37(6), 415-425, doi:10.1016/j.chiabu.2013.02.006.

Stoltenborgh, M., van Ijzendoorn, M. H., Euser, E. M., \& Bakermans-Kranenburg, M. J. (2011). A global perspective on child sexual abuse: Meta-analysis of prevalence around the world. Child Maltreatment, 16(2), 79-101, doi:http://dx.doi.org/10.1177/1077559511403920.

Swart, J., \& Apsche, J. (2014). Mindfulness, mode deactivation, and family therapy: A winning combination for treating adolescents with complex trauma and behavioural problems. International Journal of Behavioral Consultation and Therapy, 9(2), 9-14. 
Terwee, C. B., Bot, S. D., de Boer, M. R., van der Windt, D. A., Knol, D. L., Dekker, J., et al. (2007). Quality criteria were proposed for measurement properties of health status questionnaires. Journal of Clinical Epidemiology, 60(1), 34-42, doi:10.1016/j.jclinepi.2006.03.012.

Thompson, B. L., \& Waltz, J. (2010). Mindfulness and experiential avoidance as predictors of posttraumatic stress disorder avoidance symptom severity. Journal of Anxiety Disorders, 24(4), 409-415, doi:http://dx.doi.org/10.1016/j.janxdis.2010.02.005.

Tovote, K. A., Fleer, J., Snippe, E., Peeters, A. C., Emmelkamp, P. M., Sanderman, R., et al. (2014). Individual mindfulness-based cognitive therapy and cognitive behavior therapy for treating depressive symptoms in patients with diabetes: results of a randomized controlled trial. Diabetes Care, 37(9), 2427-2434, doi:10.2337/dc13-2918.

Tyler, K. A. (2002). Social and emotional outcomes of childhood sexual abuse: A review of recent research. Aggression and Violent Behavior, 7(6), 567-589, doi:http://dx.doi.org/10.1016/S1359-1789\%2801\%2900047-7.

Valdez, C., \& Lilly, M. (2015). Self-Compassion and Trauma Processing Outcomes Among Victims of Violence. Mindfulness, 1-11, doi:10.1007/s12671-015-0442-3.

van der Velden, A. M., Kuyken, W., Wattar, U., Crane, C., Pallesen, K. J., Dahlgaard, J., et al. (2015). A systematic review of mechanisms of change in mindfulness-based cognitive therapy in the treatment of recurrent major depressive disorder. Clinical Psychology Review, 37, 26-39, doi:10.1016/j.cpr.2015.02.001.

Wowra, S. A., \& McCarter, R. (1999). Validation of the Empowerment Scale with an outpatient mental health population. Psychiatric Services, 50(7), 959-961, doi:10.1176/ps.50.7.959. 
Zoogman, S., Goldberg, S. B., Hoyt, W. T., \& Miller, L. (2015). Mindfulness interventions with youth: A meta-analysis. Mindfulness, 6(2), 290-302, doi:http://dx.doi.org/10.1007/s12671-013-0260-4. 